\title{
omunidade arbórea em CerRadão na bacia do Rio Verde, Mato Grosso do SUl, Brasil
}

\author{
Ricardo Anghinoni Bocchese \\ Richard Matheus Fernandes \\ Universidade para o Desenvolvimento do Estado e da Região do Pantanal, Rua \\ Alexandre Herculano, 1400, Jardim Veraneio, 79037-280, Campo Grande, Mato \\ Grosso do Sul, Brasil. bocchese.ra@gmai.com

\section{Solon Rodrigo Vilhalba Azeredo} \\ Universidade Estadual de Mato Grosso do Sul, Rodovia Aquidauana/UEMS, Km 12, \\ 79200-000 Aquidauana, Mato Grosso do Sul, Brasil.
}

Resumo: O objetivo neste estudo foi verificar a análise fitossociológica da comunidade arbórea de cerradão em um trecho da bacia do rio Verde, Mato Grosso do Sul. Foram demarcadas 100 parcelas de $1.000 \mathrm{~m}^{2}$, com a medição das árvores com diâmetro à altura do peito (DAP) maior ou igual a 10 $\mathrm{cm}$. Foram levantados 2.638 indivíduos distribuídos um total de 91 espécies lenhosas. Tapirira guianensis obteve o maior valor de importância $(13,3 \%)$. A diversidade $\left(H^{\prime}=3,493\right)$ e a equabilidade ( $J^{\prime}$ $=0,77)$ mostraram notável distribuição ao longo da área levantada. A riqueza e a composição da amostragem, bem como os valores de Shannon e Pileou gerados, mostraram-se compatíveis com informações de outros estudos, indicando que a vegetação lenhosa amostrada na bacia do rio Verde condiz com o esperado para áreas de cerradões do Centro-Oeste.

Palavras-chave: Cerrado, comunidade arbórea, fitossociologia, savana florestada

\section{Arboreal community of a wooded savanna over Verde River banks, Mato Grosso DO SUL, BRAzIL}

\begin{abstract}
The aim in this study was to verify the phytosociological analysis of a wooded savanna of Verde River's bank stretch in Mato Grosso do Sul, Brazil. We demarcated 100 plots of $1.000 \mathrm{~m}^{2}$ where trees with a minimum diameter at breast high of $10 \mathrm{~cm}$ were measured. A total of 2.638 trees were sampled, which were distributed in 91 tree species. Tapirira guianensis obtained the highest value of importance $(13.3 \%)$. Diversity $\left(H^{\prime}=3.493\right)$ and Equability $\left(J^{\prime}=0.77\right)$ showed remarkable variation along the sampled area. Richness and composition sampling values, as well as Shannon and Pileou values generated were similar to other studies, what indicates that the sampled vegetation found in Rio Verde basin is consistent with the expected for areas of wooded savanna in Brazil's Midwest.
\end{abstract}

Keywords: Brazilian savanna, arboreal community, phytosociology, wooded savanna 


\section{INTRODUÇÃO}

Estudos sobre vegetação nativa fornecem suporte a uma gama de decisões relacionadas a processos ecológicos (Durigan, 2012). Partindo do pressuposto de que o componente arbóreo é o que sustenta primordialmente um ecossistema florestal (Couto, 2005), a verificação da composição e do arranjo da comunidade arbórea estabelecida auxilia na avaliação do status de conservação dos ecossistemas naturais. Tal fator é fundamental para o embasamento de linhas de pesquisa em ecologia e subsidiar ações de manejo, regeneração e conservação ambiental (Farinaccio et al., 2018).

Neste contexto, a fitossociologia apresenta-se como uma ferramenta importante para observar a distribuição de uma vegetação florestal nativa, onde a coleta de informações quali-quantitativas das populações permite o conhecimento de seus aspectos ecológicos ( $\mathrm{Ca}$ bacinha \& Castro, 2010; Durigan, 2012; Santos et al., 2012, Chaves et al., 2013).

A geração de informações sobre a vegetação do Cerrado é sempre essencial, tendo em vista a grande diversidade regional de espécies e os elevados índices de endemismo no bioma (Ratter et al., 1997; Borlaug, 2002; Colli et al., 2002; Sawyer, 2002). Dentre as suas variedades fitofisionômicas, o cerradão (Savana Florestada) caracteriza-se por ser um sub-grupo de formação florestal cujas árvores comumente atingem de 8 a $15 \mathrm{~m}$ de altura, composto por espécies arbóreas típicas de formações savânicas mais abertas e de mata (Oliveira-Filho \& Ratter 1995; Ribeiro \& Walter 2008).

Mato Grosso do Sul está inserido na Ecorregião Paraná Guimarães, a maior das 22 Ecorregiões do Cerrado (18,9\% da área do Bioma) (Felfili et al., 2002). Estudos desenvolvidos em fragmentos de cerradão no MS evidenciaram variações na composição das espécies dominantes em função de aspectos regionais, como estrutura do solo e a influência de outras tipologias florestais (Ratter et al.,2003; Salis et al., 2006; Camilotti et al., 2011; Solórzano et al., 2012).

A bacia do Rio Verde no MS é de grande expressão por ser um dos principais afluentes do rio Paraná (Arruda et al., 2008), fazendo divisa com os Estados de São Paulo, Goiás e Mato Grosso. A vegetação nas cabeceiras dos córregos apresenta-se predominante como cerrado típico, tendendo a cerradão em direção à foz (Lanza et al., 2014). A região é deficiente em inventários biológicos, em parte devido à precária infraestrutura viária.

O objetivo neste estudo foi conhecer a composição florística e espécies dominantes de comunidade arbórea em cerradão na bacia do rio Verde, com o intuito de gerar informações florísticas e da estrutura da vegetação regionais.

\section{Material e métodos}

Entre os meses de maio e julho de 2017 foram demarcadas 100 parcelas retangulares de $1.000 \mathrm{~m}^{2}$ (100 metros $\times 10$ metros) na microbacia do rio Verde, abrangendo os municípios de Ribas do Rio Pardo, Água Clara, Três Lagoas e Brasilândia, na porção centro-leste de Mato Grosso do Sul (Fig. 1). Como área de abrangência do estudo foi estabelecida a localização geográfica (World Geodetic System - WGS 84) do trecho contemplado desde a primeira parcela, numerada como 01 (2009'73"S; 5307'09"W) até a última parcela, numerada como 100

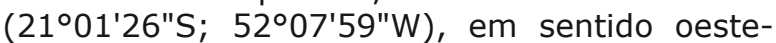
leste paralelamente ao rio Verde.

O relevo da região se caracteriza pela marcante morfoestrutura e litologia do Grupo Bauru em formas conservadas, com altimetria variando de 320 a 700 metros, predominando Latossolos e Neossolos (Pinheiro et al., 2008). O clima da região é do tipo tropical úmido (Aw) segundo a classificação de Köeppen (Alvares et al., 2014), com verões chuvosos e invernos secos, onde a média pluviométrica anual situa-se em torno de $1.500 \mathrm{~mm}$.

As parcelas foram demarcadas aleatoriamente, sendo uma por fragmento selecionado. A seleção das áreas amostrais considerou a representatividade dos fragmentos, evitando-se amostrar trechos descaracterizados ou em estágios iniciais de formação secundária. Com este tamanho de parcelas foram desconsiderados fragmentos pequenos e alterados que apresentassem evidente variação estrutural horizontal na vegetação lenhosa.

Para o levantamento fitossociológico foram considerados todos os indivíduos lenhosos vivos inseridos nas parcelas que obtiveram diâmetro à altura do peito (DAP), a $1,30 \mathrm{~m}$ do solo, maior ou igual a $10 \mathrm{~cm}$. Os parâmetros fitossociológicos (densidade, frequência, dominância e valor de importância) foram estimados segundo as proposições de Mueller-Dumbois \& Ellenberg (1974). O cálculo dos índices de diversidade de Shannon $\left(\mathrm{H}^{\prime}\right)$ e Equabilidade de Pielou ( $\mathrm{J}^{\prime}$ ) foram feitos com base logarítmica neperiana. A identificação botânica foi realizada por método visual e registro fotográfico de espécimes. A taxonomia botânica seguiu o sistema de classificação APG IV (2016).

\section{Resultados e discussão}

$\mathrm{Na}$ avaliação das 100 parcelas foram levantados 2.638 indivíduos, o que corresponde a uma densidade de 264 ind/ha ${ }^{-1}$. Em um estudo 

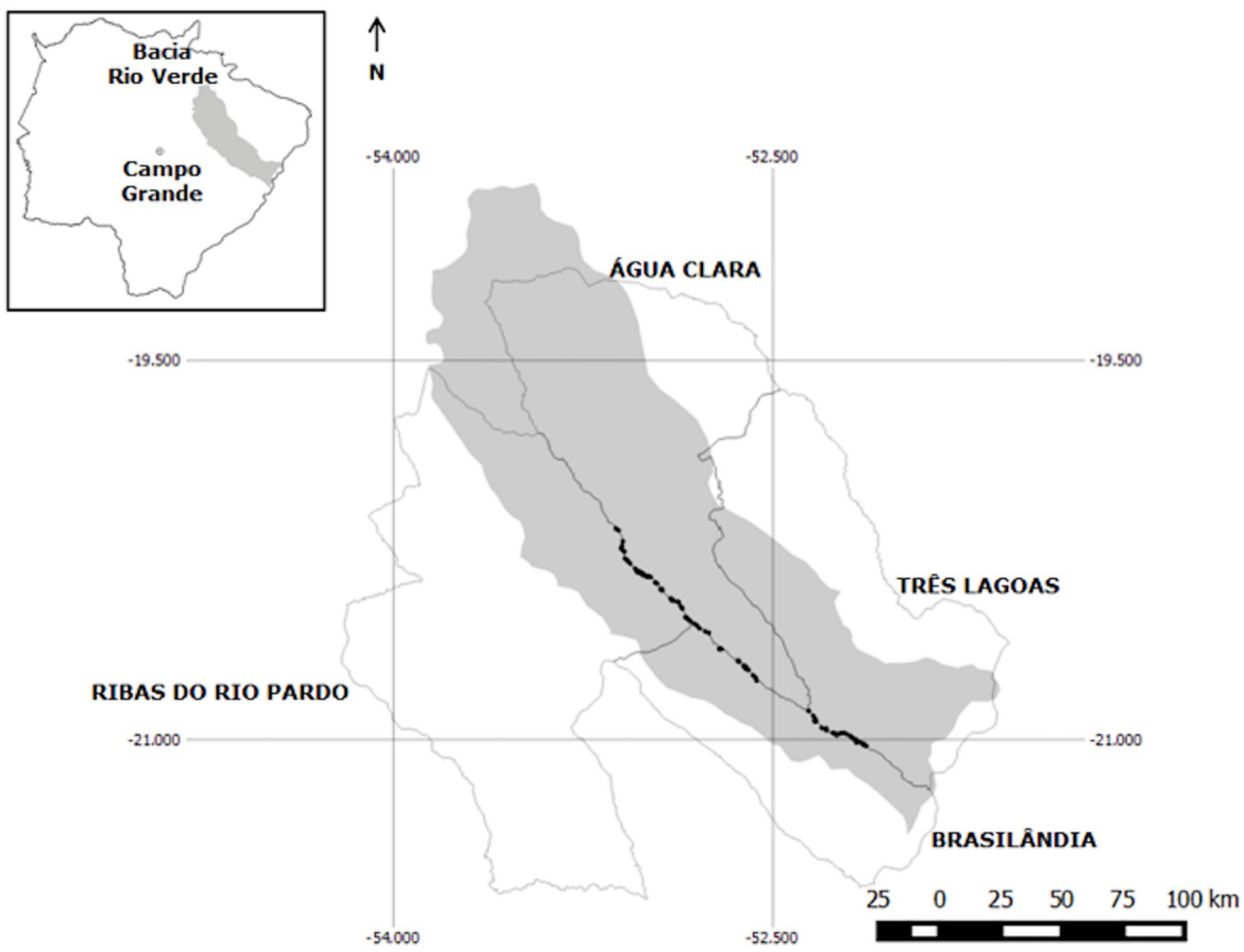

Fig. 1. Localização espacial da amostragem de vegetação de cerradão, bacia do Rio Verde, Mato Grosso do Sul, Brasil, 2017.

realizado em cerradão do Pantanal do MS, Solórzano et al. (2012) verificaram densidade aproximada, de 306 ind/ha ${ }^{-1}$, também considerando inclusão das árvores com DAP mínimo de $10 \mathrm{~cm}$. Maiores densidades podem ser verificadas quando o critério de inclusão das árvores é menor, a exemplo de outros oito levantamentos em cerradões do MS, cujas densidades variaram de 438 ind $/ \mathrm{ha}^{-1}$ a 833 ind $/ \mathrm{ha}^{-1}$ considerando DAP mínimo de 5,0 cm (Salis et al., 2006; Solórzano et al., 2012).

Foram identificadas 86 espécies distribuídas em 35 famílias, além de outras cinco morfoespécies, totalizando 91 taxa (Tab. 1). O número de espécies registrado neste estudo foi superior aos valores apresentados por Ratter et al.(2003) em cinco áreas de cerradão inseridos na bacia do Rio Verde em Mato Grosso do Sul, que abrangeram os municípios de Água Clara $(54,60,65)$, Ribas do Rio Pardo (72) e Três Lagoas (63).

Oliveira-Filho \& Ratter (1995) colocaram que a riqueza de árvores em cerradão em geral é menor do que 120 espécies. Em uma avalia- ção de 33 áreas de cerradão distribuídas em diferentes locais do bioma Cerrado, Solórzano et al. (2012) verificaram riquezas que variaram de 49 a 124 espécies. Para Guilherme et al. (2020), o número de 100 espécies é considerado uma riqueza elevada para cerradões, por esta fisionomia abranger aspectos florestais e savânicos.

As espécies aqui registradas (Tab. 1) são compatíveis com suas distribuições geográficas para Mato Grosso do Sul e são de ampla ocorrência no bioma Cerrado (Goodland, 1970; Pott \& Pott, 1994; Ratter et al., 2003; Silva Junior, 2005; Salis et al., 2006; Silva Junior \& Pereira, 2009; Solórzano et al., 2012; Bueno et al., 2018). Dos 86 taxa identificados, 39 (32\%) pertencem ao grupo das 121 espécies consideradas oligárquicas no bioma, ou seja, aquelas que possuem distribuição abrangente e que dominam a flora lenhosa do Cerrado brasileiro (Bridgewater et al., 2004) (Tab.1).

Dentre elas, estão Tapirira guianensis Aubl. e Copaifera langsdorffii Desf., que obtiveram os maiores Valores de Importância (VI) deste 
Tab. 1. Espécies lenhosas registrada no estudo fitossociológico na bacia do rio Verde, Mato Grosso do Sul, Brasil, 2017. *Espécies oligárquicas segundo Bridgewater et al. (2004).

\begin{tabular}{|c|c|}
\hline Família & Nomenclatura científica \\
\hline \multirow[t]{3}{*}{ Anacardiaceae } & Astronium fraxinifolium Schott* \\
\hline & Myracrodruon urundeuva M.Allemão \\
\hline & Tapirira guianensis Aubl.* \\
\hline \multirow[t]{5}{*}{ Annonaceae } & Duguetia furfuracea (A.St.-Hil.) Saff.*' \\
\hline & Annona coriacea Mart.* \\
\hline & Unonopsis lindmanii R.E.Fr. \\
\hline & Xylopia aromatica (Lam.) Mart.* \\
\hline & Xylopia emarginata Mart. \\
\hline Apocynaceae & Himatanthus obovatus (Müll.Arg.) Woodson* \\
\hline Araliaceae & Didymopanax macrocarpum (Cham. \& Schltdl.) Seem.* \\
\hline \multirow[t]{3}{*}{ Arecaceae } & Acrocomia aculeata (Jacq.) Lodd. ex Mart. \\
\hline & Mauritia flexuosa L.f. \\
\hline & Syagrus oleracea (Mart.) Becc. \\
\hline \multirow[t]{5}{*}{ Bignoniaceae } & Handroanthus ochraceus (Cham.) Mattos* \\
\hline & Handroanthus serratifolius (Vahl.) S.Grose \\
\hline & Jacaranda cuspidifolia Mart. \\
\hline & Tabebuia aurea (Silva Manso) Benth. \& Hook.f. ex S.Moore \\
\hline & Tabebuia heptaphylla (Vell.) Toledo \\
\hline Boraginaceae & Cordia cf. sellowiana Cham. \\
\hline Burseraceae & Protium heptaphyllum (Aubl.) Marchand* \\
\hline \multirow[t]{2}{*}{ Calophyllaceae } & Calophyllum brasiliense Cambess. \\
\hline & Kielmeyera speciosa A.St.-Hil.* \\
\hline Caryocaraceae & Caryocar brasiliense Cambess.* \\
\hline Chrysobalanaceae & Hirtella gracilipes (Hook.f.) Prance \\
\hline \multirow[t]{2}{*}{ Combretaceae } & Buchenavia tomentosa Eichler \\
\hline & Terminalia argentea Mart. \& Zucc.* \\
\hline Dilleniaceae & Curatella americana L.* \\
\hline 'Ebenaceae & Diospyros hispida A.DC.* \\
\hline \multirow[t]{3}{*}{ Euphorbiaceae } & Croton urucurana Baill. \\
\hline & Mabea fistulifera Mart. \\
\hline & Maprounea guianensis Aubl.* \\
\hline \multirow[t]{10}{*}{ Fabaceae } & Acacia cf. polyphylla DC. \\
\hline & Anadenanthera colubrina (Vell.) Brenan* \\
\hline & Anadenanthera falcata (Benth.) Speg. \\
\hline & Anadenanthera macrocarpa (Benth.) Brenan \\
\hline & Andira cujabensis Benth.* \\
\hline & Bauhinia sp. \\
\hline & Bowdichia virgilioides Kunth* \\
\hline & Copaifera langsdorffii Desf.* \\
\hline & Dipteryx alata Vogel* \\
\hline & Diptychandra aurantiaca Tul. \\
\hline
\end{tabular}


... continuação Tab. 1.

\begin{tabular}{|c|c|}
\hline Fabaceae & $\begin{array}{l}\text { Enterolobium contortisiliquum (Vell.) Morong } \\
\text { Erythrina mulungu Mart. } \\
\text { Hymenaea stigonocarpa Mart. ex Hayne* } \\
\text { Inga laurina (Sw.) Willd. } \\
\text { Inga vera Wild. } \\
\text { Lonchocarpus sp. } \\
\text { Machaerium acutifolium Vogel* } \\
\text { Machaerium sp. } \\
\text { Ormosia arborea (Vell.) Harms } \\
\text { Peltogyne confertiflora (Mart. ex Hayne) Benth. } \\
\text { Plathymenia reticulata Benth.* } \\
\text { Platypodium elegans Vogel* } \\
\text { Vatairea macrocarpa (Benth.) Ducke.* }\end{array}$ \\
\hline Lauraceae & Nectandra sp. \\
\hline Loganiaceae & Strychnos pseudoquina A.St.-Hil.* \\
\hline Lythraceae & Lafoensia pacari A.St.-Hil* \\
\hline Malpighiaceae & $\begin{array}{l}\text { Byrsonima verbascifolia (L.) DC.* } \\
\text { Byrsonima sp. }\end{array}$ \\
\hline Malvaceae & $\begin{array}{l}\text { Luehea cf. candicans Mart. \& Zucc. } \\
\text { Luehea grandiflora Mart. \& Zucc. } \\
\text { Pseudobombax longiflorum (Mart.) A.Robyns* }\end{array}$ \\
\hline Meliaceae & Trichilia pallida Sw. \\
\hline Moraceae & $\begin{array}{l}\text { Ficus insipida Wild. } \\
\text { Ficus sp. } \\
\text { Ficus cf. enormis Mart. Ex Miq. } \\
\text { Sorocea bonplandii (Baill.) W.C.Burger et al. }\end{array}$ \\
\hline Myrtaceae & $\begin{array}{l}\text { Eugenia sp. } \\
\text { Myrcia bella Cambess. } \\
\text { Myrcianthes cf. pungens (O.Berg) D. Legrand } \\
\text { Psidium sp. }\end{array}$ \\
\hline Phytolaccaceae & Gallesia integrifolia (Spreng.) Harms \\
\hline Polygonaceae & Coccoloba mollis Casar. \\
\hline Primulaceae & Rapanea gardneriana (A.DC.) Mez \\
\hline Proteaceae & Roupala montana Aubl.* \\
\hline Rhamnaceae & Rhamnidium elaeocarpum Reissek* \\
\hline Rubiaceae & Alibertia edulis (Rich.) A.Rich.* \\
\hline Rutaceae & Zanthoxylum riedelianum Engl. \\
\hline Sapindaceae & $\begin{array}{l}\text { Dilodendron bipinnatum Radlk.* } \\
\text { Magonia pubescens A.St.-Hil.* }\end{array}$ \\
\hline Sapotaceae & $\begin{array}{l}\text { Pouteria ramiflora (Mart.) Radlk.* } \\
\text { Pouteria torta (Mart.) Radlk.* }\end{array}$ \\
\hline Urticaceae & Cecropia pachystachya Trécul \\
\hline
\end{tabular}

Rev. Biol. Neotrop. / J. Neotrop. Biol., Goiânia, v. 17, n. 2, p. 91-102, jul.-dez. 2020 
... continuação Tab. 1.

\begin{tabular}{ll}
\hline Vochysiaceae & $\begin{array}{l}\text { Qualea grandiflora Mart.* } \\
\text { Qualea multiflora Mart.* } \\
\text { Vochysia haenkeana Mart.* } \\
\text { Indeterminadas } \\
\text { Morfoespécie } 1\end{array}$ \\
Morfoespécie 2 & Morfoespécie 3 \\
Morfoespécie 4 \\
Morfoespécie 5 \\
\hline
\end{tabular}

estudo (Tab.2). Juntas, contemplaram 774 indivíduos (29,5\% da abundância e $33,97 \%$ da dominância).

T. guianensis está dentre as espécies mais ocorrentes da família Anacardiaceae (Souza \& Lorenzi, 2008), distribuída em quase todo território nacional. Em função de sua ocorrência em formações secundárias com solos úmidos e

Tab. 2. Fitossociologia de 100 parcelas de $1.000 \mathrm{~m} 2$ amostradas na bacia do rio Verde, Mato Grosso do Sul, Brasil, 2017. N (número de indivíduos), DR (densidade relativa), FR (frequência relativa), DoR (dominância relativa), VI (Valor de importância).

\begin{tabular}{lccccc}
\hline \multicolumn{1}{c}{ Taxa } & N & DR & FR & DoR & VI \\
\hline Tapirira guianensis Aubl. & 477 & 18,08 & 7,82 & 13,97 & 39,87 \\
Copaifera langsdorffii Desf. & 297 & 11,26 & 7,58 & 19,85 & 38,68 \\
Buchenavia tomentosa Eichler & 121 & 4,59 & 5,25 & 8,02 & 17,86 \\
Xylopia aromatica (Lam.) Mart. & 170 & 6,44 & 6,65 & 2,67 & 15,77 \\
Anadenanthera falcata (Benth.) Speg. & 133 & 5,04 & 4,55 & 5,82 & 15,40 \\
Hymenaea stigonocarpa Mart. Ex Hayne & 45 & 1,71 & 2,57 & 4,15 & 8,42 \\
Qualea grandiflora Mart. & 76 & 2,88 & 2,43 & 2,54 & 7,75 \\
Qualea multiflora Mart. & 66 & 2,5 & 2,57 & 2,32 & 7,38 \\
Anadenanthera colubrina (Vell.) Brenan & 42 & 1,59 & 1,63 & 3,12 & 6,35 \\
Magonia pubescens A. St.-Hil. & 50 & 1,9 & 1,98 & 2,24 & 6,12 \\
Pouteria torta (Mart.) Radlk. & 56 & 2,12 & 2,1 & 1,84 & 6,06 \\
Coccoloba mollis Casar. & 61 & 2,31 & 2,45 & 1,22 & 5,98 \\
Curatella americana L. & 58 & 2,2 & 2,1 & 1,61 & 5,90 \\
Luehea grandiflora Mart. \& Zucc. & 61 & 2,31 & 1,87 & 1,38 & 5,56 \\
Ormosia arborea (Vell.) HarmsA & 59 & 2,24 & 1,52 & 1,29 & 5,05 \\
Diptychandra aurantiaca Tul. & 50 & 1,9 & 1,75 & 1,05 & 4,70 \\
Protium heptaphyllum (Aubl.) Marchand & 37 & 1,4 & 1,63 & 1,57 & 4,60 \\
Myracrodruon urundeuva M.Allemão & 41 & 1,55 & 1,28 & 1,68 & 4,53 \\
Myrcia bella Cambess. & 40 & 1,52 & 1,52 & 1,12 & 4,15 \\
Psidium sp. & 34 & 1,29 & 2,1 & 0,43 & 3,82 \\
Tabebuia heptaphylla (Vell.) Toledo & 18 & 0,68 & 1,4 & 1,64 & 3,73 \\
Jacaranda cuspidifoila Mart. & 27 & 1,02 & 1,87 & 0,74 & 3,63 \\
Terminalia argentea Mart. \& Succ. & 22 & 0,83 & 1,17 & 1,58 & 3,57 \\
Platypodium elegans Vogel & 26 & 0,99 & 1,28 & 1,18 & 3,46 \\
Zanthoxylum riedelianum Engl. & 25 & 0,95 & 1,52 & 0,93 & 3,40 \\
Dipteryx alata Vogel & 24 & 0,91 & 1,4 & 0,99 & 3,29 \\
Lonchocarpus sp. & 25 & 0,95 & 1,52 & 0,79 & 3,26 \\
\hline
\end{tabular}


... continuação Tab. 2.

\begin{tabular}{|c|c|c|c|c|c|}
\hline Annona coriacea Mart. & 29 & 1,1 & 1,05 & 0,97 & 3,11 \\
\hline Inga vera Wild. & 34 & 1,29 & 0,93 & 0,75 & 2,97 \\
\hline Nectandra sp. & 24 & 0,91 & 1,17 & 0,73 & 2,80 \\
\hline Hirtella gracilipes (Hook. f.) Prance & 22 & 0,83 & 1,4 & 0,30 & 2,54 \\
\hline Machaerium acutifolium Vogel & 20 & 0,76 & 0,82 & 0,53 & 2,10 \\
\hline Dilodendron bipinnatum Radlk. & 18 & 0,68 & 0,82 & 0,57 & 2,06 \\
\hline Calophyllum brasiliense Cambess. & 19 & 0,72 & 0,47 & 0,82 & 2,01 \\
\hline Syagrus oleracea (Mart.) Becc. & 17 & 0,64 & 0,93 & 0,39 & 1,97 \\
\hline Astronium fraxinifolium Schott & 14 & 0,53 & 0,93 & 0,50 & 1,97 \\
\hline Maprounea guianensis Aubl. & 17 & 0,64 & 0,58 & 0,66 & 1,89 \\
\hline Caryocar brasiliense Cambess. & 12 & 0,45 & 0,93 & 0,45 & 1,83 \\
\hline $\begin{array}{l}\text { Tabebuia aurea (Silva Manso) Benth. \& } \\
\text { Hook.f .ex S. Moore }\end{array}$ & 19 & 0,72 & 0,82 & 0,30 & 1,80 \\
\hline Eugenia sp. & 13 & 0,49 & 1,05 & 0,25 & 1,80 \\
\hline Andira cujabensis Benth. & 12 & 0,45 & 0,93 & 0,22 & 1,61 \\
\hline Cecropia pachystachya Trécul & 10 & 0,38 & 1,05 & 0,16 & 1,59 \\
\hline Morfoespécie 1 & 19 & 0,72 & 0,35 & 0,45 & 1,52 \\
\hline Plathymenia reticulada Benth. & 9 & 0,34 & 0,82 & 0,35 & 1,51 \\
\hline Handroanthus serratifolius (Vahl.) S.Grose & 9 & 0,34 & 0,7 & 0,22 & 1,26 \\
\hline Byrsonima verbascifolia (L.) DC. & 10 & 0,38 & 0,7 & 0,18 & 1,26 \\
\hline Alibertia edulis (Rich.) A.Rich. & 9 & 0,34 & 0,82 & 0,10 & 1,25 \\
\hline Byrsonima sp. & 9 & 0,34 & 0,7 & 0,13 & 1,17 \\
\hline Unonopsis lindmanii R.E. Fr. & 13 & 0,49 & 0,47 & 0,20 & 1,16 \\
\hline Ficus cf. enormis Mart. Ex Miq. & 4 & 0,15 & 0,47 & 0,49 & 1,11 \\
\hline Acrocomia aculeata (Jacq.) Lodd. Ex Mart. & 7 & 0,27 & 0,58 & 0,22 & 1,07 \\
\hline Rhamnidium elaeocarpum Reissek & 7 & 0,27 & 0,35 & 0,41 & 1,02 \\
\hline Diospyros hispida A.DC. & 5 & 0,19 & 0,58 & 0,17 & 0,94 \\
\hline Morfoespécie 3 & 8 & 0,3 & 0,35 & 0,25 & 0,90 \\
\hline Machaerium sp. & 5 & 0,19 & 0,47 & 0,16 & 0,81 \\
\hline Bowdichia virgilioides Kunth & 4 & 0,15 & 0,35 & 0,27 & 0,78 \\
\hline Roupala montana Aubl. & 6 & 0,23 & 0,47 & 0,07 & 0,76 \\
\hline Morfoespécie 4 & 6 & 0,23 & 0,35 & 0,14 & 0,72 \\
\hline Pseudobombax longiflorum (Mart.) A. Robyns & 2 & 0,08 & 0,23 & 0,33 & 0,64 \\
\hline Luehea cf. candicans Mart. \& Zucc. & 4 & 0,15 & 0,35 & 0,10 & 0,60 \\
\hline Anadenanthera macrocarpa (Benth.) Brenan & 5 & 0,19 & 0,23 & 0,17 & 0,59 \\
\hline Erythrina mulungu Mart. & 4 & 0,15 & 0,23 & 0,17 & 0,56 \\
\hline Ficus insipida Wild. & 3 & 0,11 & 0,35 & 0,10 & 0,56 \\
\hline Morfoespécie 2 & 7 & 0,27 & 0,12 & 0,17 & 0,56 \\
\hline Myrcianthes cf. pungens (O.Berg) D. Legrand & 4 & 0,15 & 0,23 & 0,11 & 0,49 \\
\hline Acacia cf. polyphylla DC. & 2 & 0,08 & 0,23 & 0,16 & 0,47 \\
\hline Bauhinia sp. & 4 & 0,15 & 0,23 & 0,08 & 0,46 \\
\hline Duguetia furfuracea (A.St.-Hil.) Saff. & 4 & 0,15 & 0,23 & 0,05 & 0,44 \\
\hline Lafoensia pacari A.St.-Hil. & 3 & 0,11 & 0,23 & 0,08 & 0,43 \\
\hline $\begin{array}{l}\text { Peltogyne confertiflora (Mart. ex Hayne) } \\
\text { Benth. }\end{array}$ & 3 & 0,11 & 0,23 & 0,07 & 0,41 \\
\hline Ficus sp. & 2 & 0,08 & 0,23 & 0,10 & 0,41 \\
\hline $\begin{array}{l}\text { Didymopanax macrocarpum (Cham. \& } \\
\text { Schltdl.) Seem. }\end{array}$ & 2 & 0,08 & 0,23 & 0,08 & 0,39 \\
\hline
\end{tabular}


... continuação Tab. 2.

\begin{tabular}{llllll}
\hline Morfoespécie 5 & 4 & 0,15 & 0,12 & 0,12 & 0,38 \\
Mabea fistulifera Mart. & 3 & 0,11 & 0,12 & 0,14 & 0,37 \\
Vochysia haenkeana Mart. & 2 & 0,08 & 0,23 & 0,05 & 0,36 \\
Trichilia pallida Sw. & 2 & 0,08 & 0,23 & 0,11 & 0,36 \\
Croton urucurana Baill. & 4 & 0,15 & 0,12 & 0,09 & 0,36 \\
Rapanea gardneriana (A.DC.) Mez & 2 & 0,08 & 0,23 & 0,11 & 0,35 \\
Xylopia emarginata Mart. & 4 & 0,15 & 0,12 & 0,07 & 0,33 \\
Vatairea macrocarpa (Benth.) Ducke. & 4 & 0,15 & 0,12 & 0,07 & 0,33 \\
Kielmeyera speciosa A.St.-Hil. & 2 & 0,08 & 0,23 & 0,02 & 0,33 \\
Inga laurina (Sw.) Willd. & 1 & 0,04 & 0,12 & 0,11 & 0,26 \\
Mauritia flexuosa L.f. & 1 & 0,04 & 0,12 & 0,08 & 0,24 \\
Cordia cf. sellowiana Cham. & 2 & 0,08 & 0,12 & 0,03 & 0,23 \\
Pouteria ramiflora (Mart.) Radlk. & 1 & 0,04 & 0,12 & 0,08 & 0,23 \\
Strychnos pseudoquina A.St. -Hil. & 1 & 0,04 & 0,12 & 0,05 & 0,21 \\
Handroanthus ochraceus (Cham.) Mattos & 1 & 0,04 & 0,12 & 0,04 & 0,20 \\
Gallesia integrifolia (Spreng.) Harms & 1 & 0,04 & 0,12 & 0,04 & 0,20 \\
Enterolobium contortisiliquum (Vell.) Morong & 1 & 0,04 & 0,12 & 0,02 & 0,18 \\
Himatanthus obovatus (Müll.Arg.) Woodson & 1 & 0,04 & 0,12 & 0,02 & 0,18 \\
Sorocea bonplandii (Baill.) W.C.Burger et al. & 1 & 0,04 & 0,12 & 0,01 & 0,16 \\
\hline
\end{tabular}

em ambientes secos, a espécie possui grande potencial para utilização em recuperação de áreas degradadas, sendo seus frutos amplamente apreciados pela avifauna (Lorenzi, 2016a). Já C. langsdorffii é uma das principais representantes da família Fabaceae no Brasil (Souza \& Lorenzi, 2008). Comumente presente em áreas com solos bem drenados, a espécie produz óleo com propriedades medicinais, possuindo também potencial para recuperação de áreas degradadas e na arborização urbana (Lorenzi, 2016a).

No extenso levantamento de 376 áreas do bioma Cerrado feito por Ratter et al. (2003), C. langsdorffi esteve presente em $39 \%$ delas (147), e T. guianensis, em 28\% (107). Em um estudo na própria bacia do rio Verde em Mato Grosso do Sul, Ramos et al. (2008) verificaram que a abundância de $C$. langsdorffi tendeu a aumentar com a proximidade do rio Paraná.

A família Fabaceae se destacou com 809 indivíduos e 23 espécies, correspondendo a $30,67 \%$ da abundância e $26,74 \%$ da riqueza das espécies identificadas. O predomínio desta família pode estar ligado às suas vantagens competitivas, uma vez que muitas espécies do grupo compõem a vegetação pioneira dos ecossistemas naturais (Souza \& Lorenzi, 2008; IBGE, 2012; Lorenzi, 2016ab). Estes aspectos estão ligados à alta eficiência na fixação biológica de nitrogênio, o que facilita a regeneração em solos pobres e degradados (Campello 1998), e pela capacidade de muitos representantes re- brotarem a partir de raízes (Rodrigues et al. 2004).

Fabaceae é uma das principais famílias da flora brasileira (Souza \& Lorenzi, 2008), e sua elevada representatividade também foi verificada por diversos autores em trabalhos sobre a flora de cerrados, a exemplo de Goodland (1970); Felfili \& Silva Júnior (1993); Gentry et al. (1997); Rizzini (1997); Silva et al. (2002); Ratter et al. (2003); Campos et al. (2006); Finger (2008); Mendonça et al. (2008); Bernasol \& Lima-Ribeiro (2010); Moura et al. (2010); Solórzano et al. (2012); Souza et al. (2012); Oestreich Filho (2014); Miguel et al. (2016); Silva \& Souza (2016).

Vinte famílias $(57,14 \%)$ foram representadas por apenas uma espécie cada. Quarenta espécies de menor abundância obtiveram dominância relativa inferior a $1 \%$ cada, que somadas, representaram $17,3 \%$ do total. A elevada concentração de espécies em poucas famílias botânicas foi um aspecto também evidenciado em outros trabalhos englobando cerradões (Nogueira et al., 2001; Felfili et al., 2002; Silva et al., 2002; Campos et al., 2006; Salis et al., 2006; Camilotti et al., 2011).

A condição de que o cerradão caracterizase como uma tipologia com estreitas conexões florísticas com suas fitofisionomias adjacentes (Ribeiro \& Walter, 2008; Guilherme et al. 2020) pôde ser evidenciada neste estudo. Calophyllum brasiliensis Cambess., Croton urucurana Baill., Inga laurina (Sw.) Willd., Mauritia flexuosa L.f. e 
Rapanea gardneriana (A.DC.) Mez são típicas de áreas úmidas ligadas a cursos d'água (Felfili, 2001; Silva Junior \& Pereira, 2009; Lorenzi, 2016ab). A presença destas espécies sugere uma heterogeneidade ambiental formada pela influência de faixas de transição de cerradão com matas ciliares e matas de galeria da região. Os seus baixos valores de VIs é um reflexo de suas ocorrências de forma ocasional e pontual na amostragem.

O Índice de Diversidade de Shannon $\left(\mathrm{H}^{\prime}\right)$ foi de 3,49. Solórzano et al. (2012) avaliaram 25 áreas de cerradão contemplando nove Estados brasileiros (GO, MA, MG, MS, MT, SP, PI, RO, TO) e o Distrito Federal, verificando diversidade de Shannon entre 2,92 a 4,00. Especificamente para Mato Grosso do Sul, esses autores constataram valores de 3,06 (Dois irmãos do Buriti) e 3,08 (Bandeirantes). Também em um cerradão de Bandeirantes, Camilotti et al. (2011) verificaram índice de Shannon de 3,08. Salis et al. (2006) observaram valores variando de 2,90 a 3,36 em seis cerradões no Pantanal de Aquidauana e de Corumbá.

A equabilidade de Pielou $\left(J^{\prime}\right)$ foi de 0,77 , que sugere considerável homogeneidade na distribuição das espécies na amostragem. Esse valor foi próximo àqueles encontrados em Mato Grosso do Sul por Solórzano et al. (2012) em Dois Irmãos do Buriti $(0,79)$ e em Bandeirantes $(0,78)$. Em outras 17 áreas de cerradão distribuídas em DF, MG, MT, PI e TO, os autores registraram valores que variaram de 0,40 a 0,89 . Salis et al. (2006) obtiveram valores atribuídos entre 0,80 a 0,91 nos cerradões do MS.

Observou-se que os parâmetros analisados obtiveram valores compatíveis com a literatura comparada, mesmo ao considerar as diferenças de aspectos metodológicos, como por exemplo, o critério de inclusão de medida de caule de árvores.

A vegetação lenhosa de cerradão amostrada na bacia do Rio Verde em Mato Grosso do Sul apresentou riqueza, comportamento fitossociológico e aspectos de distribuição das espécies de maneira condizente para comunidades arbóreas estabelecidas nesta fitofisionomia do Cerrado.

\section{RefERÊnCIAS}

Alvares, C. A., J. L. Stape, P. C. Sentelhas, J. L. M. Gonçalves, \& G. Sparovek. 2014. Koppen's climate classification map for Brazil. Meteorol. Z. 22(6): 711-728.

APG IV. Angiosperm Phylogeny Group. 2016. An update of the Angiosperm Phylogeny Group classification for the orders and families of flowering plants: APG IV. Bot. J. Linn. Soc. 181: 1-20.
Arruda, M. B., C. E. B. Proença, S. C. Rodrigues, R. N. Campos, R. N. Martins \& E. S. Martins. 2008. Ecorregiões, unidades de conservação e representatividade ecologica do bioma Cerrado. pp. 229-270. In: Sano, S. M., S. P. Almeida \& J. F. Ribeiro (Eds.). Cerrado: ecologia e flora. Brasília, Embrapa Cerrados.

Bernasol, W. P. \& M. S. Lima-Ribeiro. 2010. Estrutura espacial e diamétrica de espécies arbóreas e seus condicionantes em um fragmento de cerrado sentido restrito no sudoeste goiano. Hoehnea. 37(2): 181198.

Borlaug, N. E. 2002. Feeding a world of 10 billion people: the miracle ahead. pp. 29-60. In: Bailey, R. (Ed.). Global warming and other eco-myths. Roseville, Competitive Enterprise Institute.

Bridgewater, S., J. A. Ratter, \& J. F. Ribeiro. 2004. Biogeographic patterns, beta-diversity and dominance in the Cerrado biome of Brazil. Biodivers Conserv. 13: 2295-2318.

Bueno, M. L., A. T. Oliveira-Filho, V. Pontara, A. Pott \& G. A. Damasceno-Júnior. 2018. Flora arbórea do Cerrado de Mato Grosso do Sul. Iheringia, Sér. Bot. 73(supl.): 53-64.

Camilotti, D. C., T. C. S. Pagotto \& A. C. Araujo. 2011. Análise da vegetação arbórea de um remanescente de Cerradão em Bandeirantes, Mato Grosso do Sul, Brasil. Iheringia, Sér. Bot. 66(1): 31-46.

Campello, E. F. C. 1998. Sucessão vegetal na recuperação de áreas degradadas. pp. 183196. In: Dias, L., E. Dias \& L. W. V. Mello (Eds.). Recuperação de áreas degradadas. Viçosa, SOBRADE.

Campos, É. P., T. G. Duarte, A. V. Neri, A. F. Silva, J. A. A, Meira Neto \& G. E. Valente. 2006. Composição florística de um trecho de cerradão e cerrado sensu stricto e sua relação com o solo na Floresta Nacional de Paraopeba, MG, Brasil. Rev. Árvore. 30(6): 471-479.

Chaves, A. C. G., R. M. S. Santos, J. O. Santos, A. A. Fernandes \& P. B. Maracajá. 2013. A importância dos levantamentos florístico e fitossociológico para a conservação e preservação das florestas. Agrop. Cientif. Semiárido. 9(2): 42-48. 
Colli, G. R., R. P. Bastos, \& A. F. B. Araújo. 2002. The character and dynamics of the Cerrado Herpetofauna. pp. 223-241. In: Oliveira, P. S. \& R. J. Marquis (Eds.).The Cerrados of Brazil: Ecology and Natural History of a Neotropical Savanna. New York, University Press.

Couto, H. T. Z. 2005. Métodos de inventário da biodiversidade de espécies arbóreas: Relatório Final de Projeto temático. Piracicaba, ESALQ/ FAPESP - Programa Biota.

Durigan, G. 2012. Estrutura e Diversidade de Comunidades Florestais. pp. 294-325. In: Martins, S. V. (Ed). Ecologia de Florestas tropicais do Brasil. 2 ed. Viçosa, Editora UFV.

Farinaccio, M. A., F. O. Roque, G. Graciolli, P. R. Souza, \& J. O. P. Pinto. 2018. A flora no Biota-MS: montando o quebra-cabeça da biodiversidade de Mato Grosso do Sul. Iheringia, Sér. Bot. 73(supl.): 11-17.

Felfili, J. M. \& M. C. Silva Júnior. 1993. A comparative study of cerrado (stricto sensu) vegetation in Central Brazil. J. Trop. Ecol. 9: 277-289.

Felfili, J. M. 2001. Distribuição de diâmetros de quatro áreas de cerrado sensu stricto na Chapada do Espigão Mestre do São Francisco. pp. 57-61. In: Felfili, J. M. \& M. C. Silva Júnior (Orgs.). Biogeografia do bioma cerrado: estudo fitofisionômico na Chapada do Espigão Mestre do São Francisco. Brasília, Universidade de Brasília, Faculdade de Tecnologia, Departamento de Engenharia Florestal.

Felfili, J. M. 2002. Padrões de diversidade do cerrado do Centro-Oeste brasileiro. pp. 5861. In: Araújo, E. L., A. N. Moura, E. S. B. Sampaio, L. M. S. Gestinari \& J. M. T. Carneiro (Eds.). Biodiversidade, conservação e uso sustentável da flora do Brasil. Recife, UFRPE.

Felfili, J. M., P. E. Nogueira, M. C. Silva Júnior, B. S. Marimon \& W. B. C. Delitti. 2002. Composição florística do cerrado sentido restrito no município de Água Boa, MT. Acta Bot. Bras. 16: 103-112.

Finger, Z. 2008. Fitossociologia de comunidades arbóreas em Savanas do Brasil Central. Tese de Doutorado. Santa Maria, UFSM.
Gentry, A. H., O. Herrera-Mac Brybe, O. Huber, B. W. Nelson \& C. B. Villamil. 1997. Regional overview: South America. pp. 269-307. In: Heiwood, V. H. \& S. D. Davis (Eds.). Centre of plant diversity. Cambridge, WWF/IUCN.

Goodland, R. J. A. 1970 . Plants of the cerrado vegetation of Brazil. Phytologia. 20(1): 5778.

Guilherme, F. A. G., G. E. Silva, C. P. CoeIho, J. D. L. Rocha \& K. Ressel. 2020. Estrutura arbórea em um cerradão no sul do Estado de Goiás. Enciclopédia Biosfera. 17(32): 318-328.

IBGE. Instituto Brasileiro de Geografia e Estatística. 2012. Manual técnico da vegetação brasileira. Rio de Janeiro.

Lanza, D. A., A. Pott, \& J. S. V. Silva. 2014. Vegetação e uso da Terra na Unidade de Planejamento e Gestão Rio Verde, Mato Grosso do Sul. Rev. GeoPantanal. 16: 251262.

Lorenzi, H. 2016a. Árvores brasileiras: manual de identificação e cultivo de plantas arbóreas nativas do Brasil. v. 1, 7 ed., Nova Odessa, Instituto Plantarum de Estudos da Flora.

Lorenzi, H. 2016b. Árvores brasileiras: manual de identificação e cultivo de plantas arbóreas nativas do Brasil. v. 2, 7 ed., Nova Odessa, Instituto Plantarum de Estudos da Flora.

Mendonça, R. C., J. M. Felfili, B. M. T. Walter, M. C. Silva Júnior, A. V. Rezende, T. S. Filgueiras, P. E. Nogueira \& C. W. Fagg. 2008. Flora vascular do bioma cerrado: checklist com 12.356 espécies. pp. 423-442. In: Sano, S. M., S. P. Almeida \& J. F. Ribeiro (Eds.). Cerrado: ecologia e flora. v. 2. Brasília, Embrapa Informação Tecnológica; Planaltina, Embrapa Cerrados.

Miguel, E. P., A. V. Rezende, F. A. Leal, R. S. Pereira \& R. R. Melo. 2016. Floristicstructural characterization and successional group of tree species in the Cerrado biome of Tocantins state, Brazil. Rev. Caatinga. 29(2): 393-404. 
Moura, I. O., V. L. Gomes-Klein, J. M. Felfili \& H. D. Ferreira. 2010. Diversidade e estrutura comunitária de cerrado sensu stricto em afloramentos rochosos no Parque Estadual dos Pirineus, Goiás. Rev. Bras. Bot. 33(3): 455-467.

Muller-Dombois, D. \& H. Ellenberg. 1974. Aims and methods on vegetation ecology. New York, John Wiley and Sons.

Nogueira, P. E., J. M. Felfili, M. C. Silva Junior, W. Delitti \& A. Sevilha. 2001. Composição florística e fitossociologia de um cerrado sentido restrito no município de Canarana, MT. Bol. do Herb. Ezequias Paulo Heringer. 8: 28-43.

Oestreich Filho, E. 2014. Fitossociologia, diversidade e similaridade entre fragmentos de cerrado stricto sensu sobre neossolos quartzarênicos órticos, nos municípios de Cuiabá e Chapada dos Guimarães, Estado de Mato Grosso, Brasil. 88 f. Dissertação (Mestrado em Ciências Florestais e Ambientais), Universidade Federal de Mato Grosso, Cuiabá.

$\overline{101}$ Oliveira-filho, A. T. \& J. A. Ratter. 1995. A study of the origin of central Brazilian forests by the analysis of plant species distribution patterns. Edinb. J. Bot. 52(2): 141-194.

Pinheiro, L. C. S. J., A. S. Castro, \& E. S. Martins. 2008. Levantamento das classes de solo existentes nas Ecorregiões inseridas no limite do cerrado continuo, 6 p. In: Faleiro,F. G. (Cord.). Planaltina, Anais do IX simpósio nacional do Cerrado - II Simpósio Internacional Savanas Tropicais.

Pott, A. \& V. L. Pott. 1994. Plantas do Pantanal. Corumbá, Centro Brasileiro de Pesquisa Agropecuária.

Ramos, V. S., G. Durigan, G. A. D. C. Franco, M. F. Siqueira \& R. R. Rodrigues. 2008. Árvores da Floresta Estacional Semidecidual: Guia de identificação de espécies. São Paulo, EDUSP.

Ratter, J. A., J. F. Ribeiro \& S. Bridgewater. 1997. The brazilian cerrado vegetation and threats to its biodiversity. Ann. Bot. 80: 223-230.
Ratter, J. A., S. Bridgewater \& J. F. Ribeiro. 2003. Analysis of the floristic composition of the Brazilian cerrado vegetation III: comparison of the woody vegetation of 376 areas. Edinb. J. Bot. 60(1): 57-109.

Ribeiro, J. F. \& B. M. T. Walter. 2008. As principais fitofisionomias do Bioma Cerrado. pp. 151-212. In: Sano, S. M., S. P. Almeida \& J. F. Ribeiro (Eds.). Cerrado: ecologia e flora. Planaltina, Embrapa Cerrados.

Rizzini, C. T. 1997. Tratado de fitogeografia do Brasil. 2 ed., Rio de Janeiro, Âmbito Cultural Edições Ltda.

Rodrigues, R. R., R. B. Torres, L. A. F. Mattes \& A. S. Penha. 2004. Tree species sprouting from root buds in a semideciduous forest affected by fires. Braz. Arch. Biol. Techn. 47(1): 127- 133.

Salis, S. M., M. A. Assis, S. M. A. Crispim \& J. C. Casagrande. 2006. Distribuição e abundância de espécies arbóreas em cerradões no Pantanal, estado do Mato Grosso do Sul, Brasil. Rev. Bras. Bot. 29(3): 339352.

Santos, M. F., H. Serafim \& P. T. Sano. 2012. Composição e estrutura arbórea em floresta estacional semidecidual no Espinhaço Meridional (Serra do Cipó, MG). Rodriguésia. 63(4): 985-997.

Sawyer, D. 2002. População, meio ambiente e desenvolvimento sustentável no cerrado. pp. 279-299. In: Hogan, D. J., R. L. Carmo, J. M. P. Cunha \& R. Baeninger (Orgs.). Migração e ambiente no Centro-Oeste. Campinas, NEPO/UNICAMP, PRONEX.

Silva, L. O., D. A. Costa, K. E. Santo Filho, H. D. Ferreira \& F. Brandão. 2002. Levantamento florístico e fitossociológico em duas áreas de cerrado sensu stricto no Parque Estadual da Serra de Caldas Novas. Acta Bot. Bras. 16(1): 43-53.

Silva, G. O. \& P. B. Souza. 2016. Fitossociologia e estrutura diamétrica de um fragmento de cerrado sensu stricto, Gurupi - TO. Desafios. 3(Especial): 22-29. 
Silva Junior, M. C. 2005. 100 árvores do Cerrado. Guia de campo. Brasília, Ed. Rede de Sementes do Cerrado.

Silva Junior, M. C. \& B. A. S. Pereira. 2009. +100 árvores do Cerrado - matas de galeria. Brasília, Ed. Rede de sementes do Cerrado.

Solórzano, A., J. R. R. Pinto, J. M. Felfili \& J. V. Hay. 2012. Perfil florístico e estrutural do componente lenhoso em seis áreas de cerradão ao longo do bioma Cerrado. Acta Bot. Bras. 26 (2): 328-341.

Souza, V. C. \& H. Lorenzi. 2008. Botânica Sistemática - Guia ilustrado para identificação das famílias de fanerógamas nativas e exóticas no Brasil, baseado em APG II. 2 ed., Nova Odessa, Instituto Plantarum de Estudos da Flora.

Souza, P. B., A. L. Souza \& J. A. A. Meira Neto. 2012. Estrutura diamétrica dos estratos e grupos ecológicos de uma área de Floresta Estacional Semidecidual, em Dionísio, MG. Rev. Árvore. 36(1): 151-160. 